\title{
Primary Debulking Surgery for Stage III Epithelial Ovarian Cancer Has a Better Outcome Than Neoadjuvant Chemotherapy Followed by Interval Debulking Surgery
}

\author{
Anwar Tawfik Amin', Badawy M. Ahmed1, Ahmed Refaat'2, Sileem Ahmed Sileem³, \\ Salah Mabrouk Khallaf ${ }^{*}$
}

${ }^{1}$ Surgical Oncology Department, South Egypt Cancer Institute; Assiut University, Assiut, Egypt

${ }^{2}$ Medical Oncology Department, South Egypt Cancer Institute; Assiut University, Assiut, Egypt

${ }^{3}$ Department of Obstetrics and Gynecology, Al-Alzhar University, Assiut, Egypt

Email: *salahmab76@yahoo.com

How to cite this paper: Amin, A.T., Ahmed, B.M., Refaat, A., Sileem, S.A. and Khallaf, S.M. (2020) Primary Debulking Surgery for Stage III Epithelial Ovarian Cancer Has a Better Outcome Than Neoadjuvant Chemotherapy Followed by Interval Debulking Surgery. Journal of Cancer Therapy, 11, 142-153.

https://doi.org/10.4236/jct.2020.113012

Received: February 25, 2020

Accepted: March 22, 2020

Published: March 25, 2020

Copyright $\odot 2020$ by author(s) and Scientific Research Publishing Inc. This work is licensed under the Creative Commons Attribution International License (CC BY 4.0).

http://creativecommons.org/licenses/by/4.0/

\section{(c) (i) Open Access}

\begin{abstract}
Background: Ovarian cancer (OC) is the most lethal gynecologic malignancy. About $70 \%$ of ovarian cancer patients have advanced disease and often not totally resectable. Previous studies of neoadjuvant chemotherapy (NACT) followed by interval debulking surgery (IDS) versus primary debulking surgery (PDS) give inconsistent results. The aim of this retrospective study is to evaluate the outcome of the neoadjuvant chemotherapy followed by IDS versus PDS followed by adjuvant chemotherapy for the International Federation of Gynecology and Obstetrics (FIGO) stage III epithelial ovarian cancer. Methods: This study was performed on eligible patients with ovarian cancer admitted in Surgical Oncology and Medical Oncology departments at South Egypt Cancer Institute-Assiut University in the period from January 2010 to December 2015. Patients were divided into two groups, the first group included those who have NACT and underwent IDS plus adjuvant chemotherapy, and the second group included those who underwent PDS followed by adjuvant chemotherapy. Results: This study included 380 cases. One hundred and fifty-four patients (40.53\%) had IDS. The remaining two hundred and twenty-six patients (59.47\%) underwent PDS. In this study, treatment modality was significant for both disease-free survival (DFS) and overall survival (OS). DFS was significantly reduced after IDS when compared to PDS (median DFS: 33.00 months vs. 45.00 months, respectively; $\mathrm{p}<0.001$ ). Also, OS was significantly reduced after IDS when compared to PDS. (Median OS: 43 months vs. 46 months, respectively; $\mathrm{p}=0.047)$. Moreover, this drop of the
\end{abstract}


survivals mainly occurred in specific subgroups such as the elderly patients, patients with bad performance status, suboptimal cytoreduction, as well as high-grade tumors. Conclusion: This study showed that PDS resulted in a better disease-free survival and overall survival than IDS. Moreover, OS and DFS have significantly dropped in specific patients' subgroups. Therefore, patients selection should be considered.

\section{Keywords}

Primary Debunking Surgery, Interval Debunking Surgery, Stage III Ovarian Cancer, Neoadjuvant Chemotherapy

\section{Introduction}

Ovarian cancer (OC) is the fifth most common cause of cancer-related death among women [1]. Of newly diagnosed ovarian cancer patients, about $70 \%$ are at the advanced stage because of the lack of symptoms in the early stages of ovarian cancer. The tumors at this stage are mostly not totally resectable. Previously, patients with ovarian cancer had a bad prognosis, with a 5-year survival was about $35 \%$. Recently, survival has been improved due to improvements in diagnosis, surgery, and chemotherapy [2].

Primary surgical treatment of ovarian cancer may have some advantages in the fields of diagnosis and staging, as well as tumor debulking, which is most clear in the International Federation of Gynecology and Obstetrics (FIGO) stage III epithelial ovarian cancer [3] [4] [5]. However, surgery alone will not cure the disease because of its widespread at the time of diagnosis. On the other hand, in advanced disease, neoadjuvant chemotherapy (NACT) may increase the chance of surgical debulking and decrease surgical morbidity and mortality [6]. The results from previous studies comparing the NACT followed by interval debulking surgery (IDS) versus primary debulking surgery (PDS) are inconsistent. Several retrospective studies concluded that there was no difference in disease-free survival (DFS) or overall survival (OS) for patients with advanced ovarian cancer treated with IDS in comparison to PDS [7] [8] [9] [10] [11]. Moreover, Bristow and Chi published a meta-analysis that involved more than 800 patients, showed that NACT compared with PDS was associated with a worse OS, and they suggested that the definitive operative intervention should be performed as early in the treatment program as possible [12]. However, a more recent meta-analysis of multiple central randomized trials concluded that survival was similar in patients treated with NACT followed by interval debulking surgery compared to primary debulking followed by chemotherapy, and they even criticized Bristow and Chi meta-analysis [13] [14]. Therefore, the aim of this retrospective study is to evaluate the outcome of IDS after primary NACT compared to PDS followed by adjuvant chemotherapy for FIGO stage III epithelial ovarian cancer. 


\section{Patient and Methods}

\subsection{Patients}

This is a retrospective study performed on all ovarian cancer cases who were admitted to Surgical Oncology and Medical Oncology Departments at South Egypt Cancer Institute, Assiut University in the period from January 2010 to December 2015. The patients' flow chart is shown in Figure 1 of the CONSORT diagram. In this period, all females aged 18 years or more, diagnosed as stage III (according to FIGO ovarian cancer staging) epithelial ovarian cancer and underwent surgical treatment, either primary or after NACT, had been included in the study. Patients with incomplete data or those who did not complete the NACT were excluded from the study. Patients lost to follow up were considered deaths. The patients were categorized according to their age, performance status, tumor grade, and the extend of cytoreduction or status of optimal debulking.

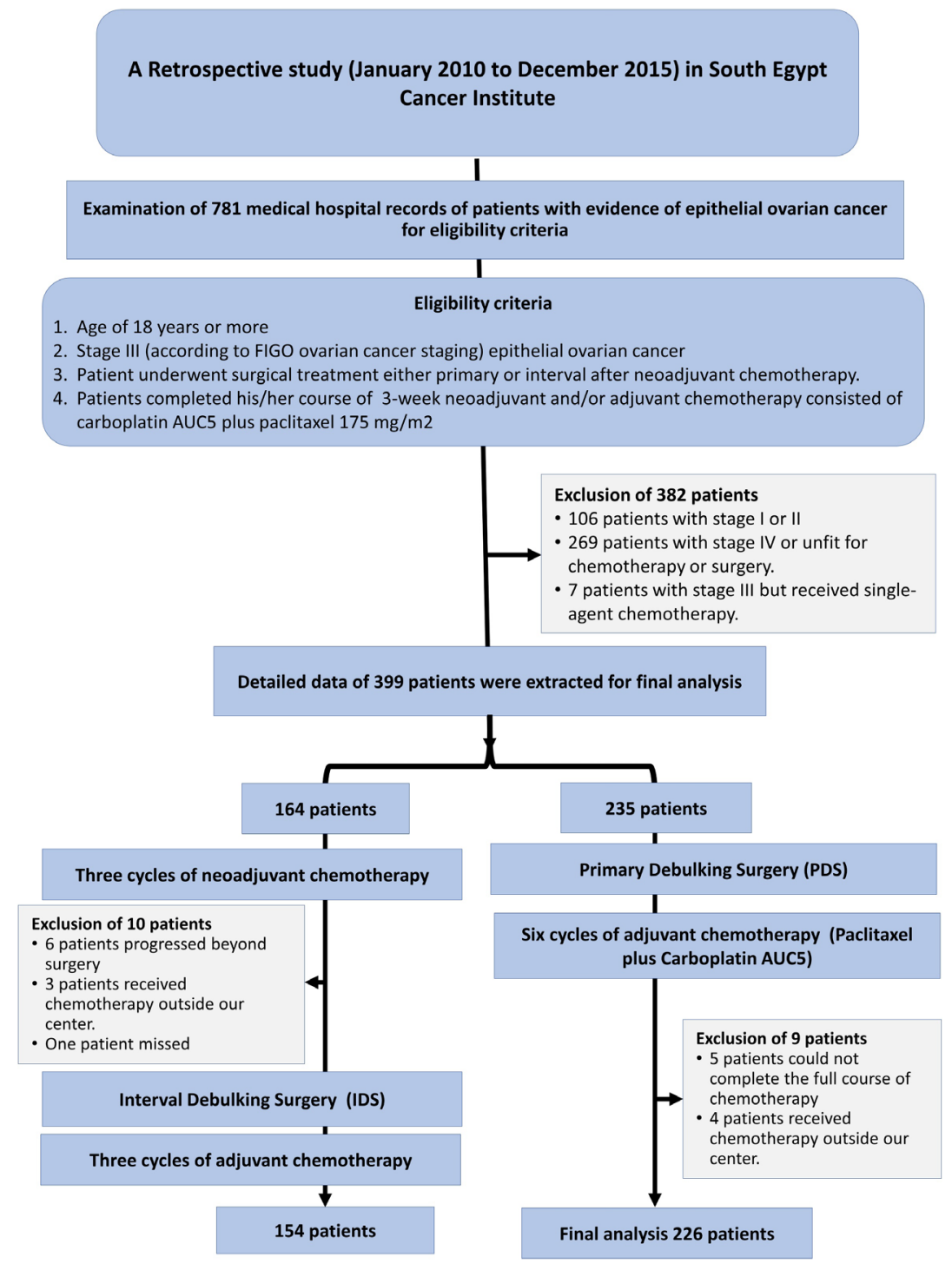

Figure 1. CONSORT diagram. 


\subsection{Treatment and Evaluation}

All patients were evaluated properly for accurate staging and underwent all routine laboratory and formal diagnostic radiological investigations. Patients were divided into two groups. The first group included patients who received three cycles of NACT, then IDS, then three additional cycles of adjuvant chemotherapy. The second group included those who have PDS, followed by six cycles of chemotherapy. The chemotherapy was given as a 3-week cycle consisted of carboplatin in a dose of the area under the curve 5 (AUC5) plus paclitaxel 175 $\mathrm{mg} / \mathrm{m}^{2}$. We collected patients' data, including demographic data, performance status, histopathological tumor grade, chemotherapy, postoperative residue, recurrences, and deaths.

\subsection{Statistical Analysis}

Statistical analysis was performed by SPSS software (Chicago, IL, US) for Windows version 21, applying parametric and non-parametric tests when appropriate. We tested the approximately normal distribution of sample through the Shapiro-Wilk's test and the visual inspection of the histograms. Categorical variables have been expressed as percentages and analyzed through the $\chi^{2}$ test or the Fisher's exact test, when appropriate. Statistically significant differences between treatment arms were defined as a p-value less than 0.05 .

DFS was defined as the time interval from the date of diagnosis to the date of the documented recurrence of the disease. If there was no documented recurrence, DFS was calculated from the date of diagnosis to the date of the last follow-up or death, whichever occurred first. OS was defined from the diagnosis date to the death date or last follow-up date.

The Kaplan-Meier method was used to estimate survival curves. For each variable, the significance of the difference in the unadjusted survival curves was assessed using the log-rank test.

We calculated hazards ratios (HRs) for survival over the entire follow-up period using a Cox proportional hazards model and 95\% confidence intervals (CIs). We entered the prognostic factors in the multivariable model to test their independent significance.

\section{Results}

\subsection{Demographics and Characteristics of the Patients}

During a period of 5 years (January 2010-December 2015), a total of three hundred eighty patients with stage III ovarian cancer met the inclusion criteria of this study, as illustrated in the CONSORT diagram. Of those, two hundred and twenty-six patients (59.47\%) underwent PDS followed by six cycles of adjuvant chemotherapy while one hundred and fifty-four (40.53\%) patients received three cycles of NACT followed by IDS plus three additional cycles of adjuvant chemotherapy (Table 1). Baseline characteristics were balanced between groups. The majority of the patients had a good performance status $(92.9 \%$ and $88.9 \%$ in 
Table 1. Demographics and baseline characteristics of enrolled patients $(\mathrm{N}=380)$ underwent primary or interval debulking surgery.

\begin{tabular}{|c|c|c|c|c|}
\hline \multirow[t]{2}{*}{ Characteristic } & \multicolumn{2}{|c|}{$\begin{array}{l}\text { Primary Debulking Surgery } \\
\qquad(\mathrm{n}=226)\end{array}$} & \multicolumn{2}{|c|}{$\begin{array}{l}\text { Interval Debulking Surgery } \\
\qquad(\mathrm{n}=154)\end{array}$} \\
\hline & No & $\%$ & No & $\%$ \\
\hline \multicolumn{5}{|l|}{ Age, Years } \\
\hline Median (Range) & \multicolumn{2}{|c|}{$55(40-82)$} & \multicolumn{2}{|c|}{$55(40-85)$} \\
\hline$<60$ & 140 & 61.9 & 105 & 68.2 \\
\hline$\geq 60$ & 86 & 38.1 & 49 & 31.8 \\
\hline \multicolumn{5}{|l|}{ ECOG PS } \\
\hline $0 / 1$ & 210 & 92.9 & 137 & 88.9 \\
\hline 2 & 16 & 7.1 & 17 & 11.1 \\
\hline \multicolumn{5}{|l|}{ Tumor Grade } \\
\hline Grade I or II & 105 & 46.5 & 77 & 50 \\
\hline Grade III & 121 & 53.5 & 77 & 50 \\
\hline \multicolumn{5}{|l|}{ Cytoreduction } \\
\hline Optimal & 136 & 60.2 & 101 & 65.6 \\
\hline Suboptimal & 90 & 39.8 & 53 & 34.4 \\
\hline
\end{tabular}

Abbreviations: ECOG PS, Eastern Cooperative Oncology Group performance status.

the PDS and IDS arms, respectively). Also, grade III tumors were present in 50\% of the patients in IDS arm, as well as in 53.5\% in PDS arm. Optimal debulking was achieved in 136 patients (60.2\%) who underwent primary surgery, while this occurred in 101 patients (65.6\%) who underwent IDS after neoadjuvant chemotherapy (Table 1).

\subsection{Disease-Free Survival}

Univariate analysis revealed that the DFS was significantly reduced after IDS when compared to PDS (median DFS: 33.00 months; 95\% CI 30.23 - 35.77 vs. 45.00 months; 95\% CI 41.25 - 48.75, respectively; $\mathrm{p}<0.001$ ) (Figure 2 and Table 2).

Also, there was a statistically significant effect of performance status of the patient on DFS, in favor of PS 0/I (median DFS: 41.00 months; 95\% CI 48.69 43.31 vs. 8 months; $95 \%$ CI 6.39 - 9.61, for patients with PS 0/I vs. those with PS II, respectively; $\mathrm{p}<0.001$ ) (Table 2). Moreover, optimal debulking surgery was associated with higher DFS when compared to suboptimal debulking (median DFS: 45.00 months; $95 \%$ CI 41.10 - 48.91 for patients underwent optimal debulking vs. 29.00 months; 95\% CI 26.77 - 31.23 for those underwent optimal debulking; $\mathrm{p}<0.001$ ) (Table 2). Moreover, there was a significant result regarding the effect of the age of the patient $(\mathrm{p}<0.001)$ and the grade of the tumor $(\mathrm{p}=$ $0.05)$ on DFS; the details are shown in Table 2. 
A. T. Amin et al.

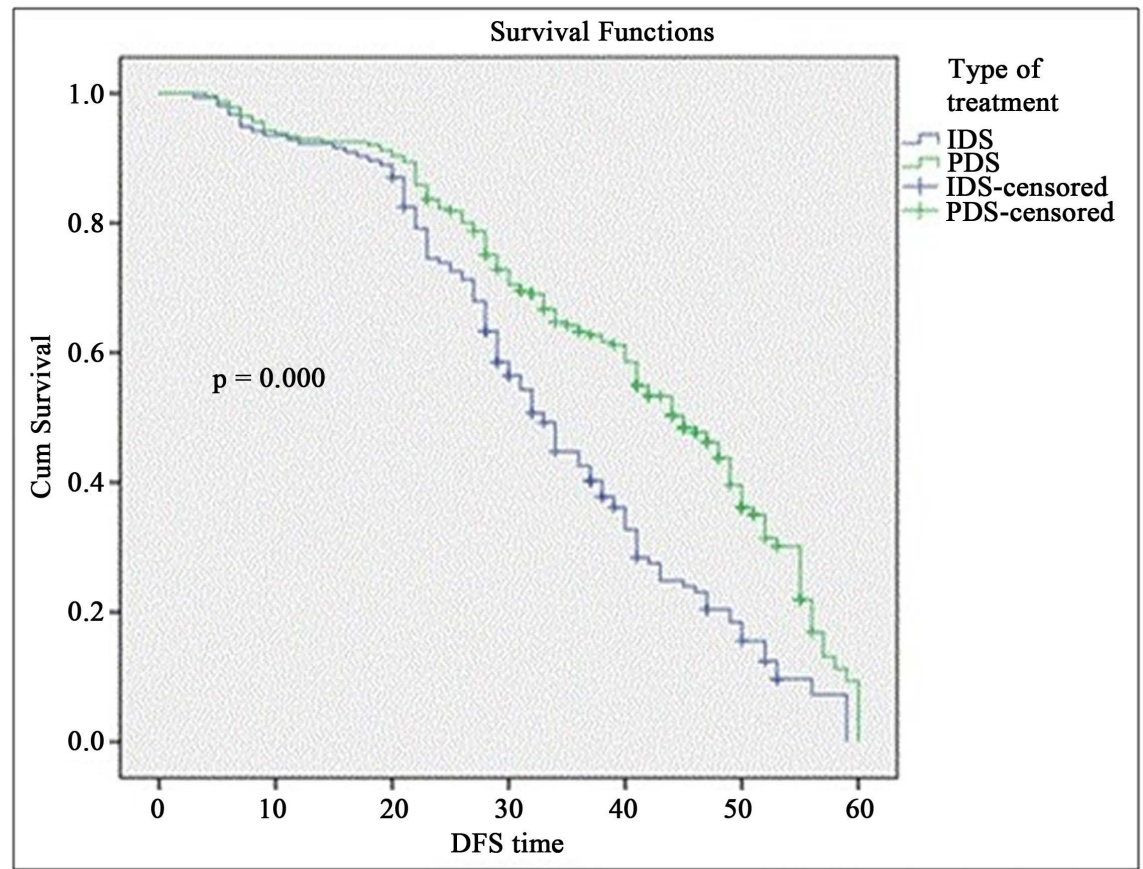

Figure 2. Kaplan-Meier analysis of DFS in patients with stage III epithelial ovarian cancer.

Table 2. Univariate and multivariate analysis of disease-free survival.

\begin{tabular}{|c|c|c|c|c|c|}
\hline \multirow{2}{*}{ Variable } & Median DFS & p Value & $\mathrm{HR}$ & $\mathrm{p}$ Value & $95 \%$ CI \\
\hline & \multicolumn{2}{|c|}{ Univariate Analysis of DFS } & \multicolumn{3}{|c|}{ Multivariate Analysis DFS } \\
\hline \multicolumn{6}{|l|}{ Age } \\
\hline $\begin{array}{l}\leq 60 \\
\geq 60\end{array}$ & $\begin{array}{l}50.2 \\
41.0\end{array}$ & $\mathrm{p}<0.001$ & 1.301 & 0.159 & $0.902-1.877$ \\
\hline \multicolumn{6}{|l|}{ PS } \\
\hline $\begin{array}{l}\text { 0/I } \\
\text { II }\end{array}$ & $\begin{array}{l}41.0 \\
8.0\end{array}$ & $\mathrm{p}<0.001$ & 0.537 & 0.003 & $0.357-0.810$ \\
\hline \multicolumn{6}{|l|}{ Treatment } \\
\hline $\begin{array}{l}\text { PDS } \\
\text { IDS }\end{array}$ & $\begin{array}{l}45.0 \\
33.0\end{array}$ & 0.01 & 0.537 & 0.03 & $0.357-0.810$ \\
\hline \multicolumn{6}{|l|}{ Cytoreduction } \\
\hline $\begin{array}{c}\text { Optimal } \\
\text { Suboptimal }\end{array}$ & $\begin{array}{l}45.0 \\
29.0\end{array}$ & $\mathrm{p}<0.001$ & 0.023 & 0.05 & $0.007-0.073$ \\
\hline \multicolumn{6}{|l|}{ Grades } \\
\hline $\mathrm{I} / \mathrm{II}$ & $\begin{array}{l}53.4 \\
41.4\end{array}$ & 0.05 & 0.116 & 0.05 & $0.040-0.334$ \\
\hline
\end{tabular}

Abbreviations: CI; confidence interval; DFS, disease-free survival; HR, hazards ratio; IDS, interval debulking surgery; PDS, primary debulking surgery; PS, performance status. 
With multivariate analysis, the type of treatment was still significant. This indicated that it was an independent prognostic factor for DFS as primary debulking surgery was associated with about $50 \%$ reduction in the risk of disease recurrence with hazards ratio (HR) of 0.53 ; 95\% CI $0.36-0.81 ; \mathrm{p}=0.03$ (Table 2, Table 3). With subgroup analysis, all patients' subgroups more benefited from PDS for DFS, except patients with PS II who achieved higher DFS with IDS ( $\mathrm{p}=$ 0.007). The details of the subgroup analysis are shown in Table 4.

\subsection{Overall Survival}

In this study, treatment modality was significant for overall survival (OS), which was significantly reduced after IDS compared to PDS. (median OS: 43 months; 95\% CI 39.46 - 46.54 vs. 46 months; 95\% CI 44.24 - 47.76, respectively; p = 0.047 ) (Figure 3 and Table 3 ). Also, the PS II, older age, and the high-grade tumor had an adverse effect on OS ( $\mathrm{p}<0.001, \mathrm{p}=0.02$, and $\mathrm{p}<0.001$, respectively) (Table 3).

With multivariate analysis, the type of treatment appeared as an independent prognostic factor for OS. Hazards ratio for this effect was 1.5; 95\% CI 1.10 - 1.70; $\mathrm{p}=0.017$, in favoring PDS (Table 3 ). With subgroup analysis, the patients that more benefited from PDS for OS were those with age of less than 60 years old ( $\mathrm{p}=$ $0.04)$, grade I/II $(\mathrm{p}=0.000)$, and PS $0 / \mathrm{I}(\mathrm{p}=0.009)$ (Table 4$)$. The only subgroup that got more benefit in OS from IDS were patients with performance status II (OS of IDS/PDS for patients with PS $=$ II was 33.7/16 months $(\mathrm{p}=0.000)$ (Table 4).

Table 3. Univariate and multivariate analysis of overall survival.

\begin{tabular}{|c|c|c|c|c|c|}
\hline \multirow{2}{*}{ Variable } & Median OS (Months) & p Value & HR & p Value & $95 \%$ CI \\
\hline & \multicolumn{2}{|c|}{ Univariate Analysis } & \multicolumn{3}{|c|}{ Multivariate Analysis } \\
\hline \multicolumn{6}{|l|}{ Age } \\
\hline$\leq 60$ & 50.5 & \multirow{2}{*}{0.02} & \multirow{2}{*}{1.301} & \multirow{2}{*}{0.17} & \multirow{2}{*}{$0.902-1.77$} \\
\hline$\geq 60$ & 45.0 & & & & \\
\hline \multicolumn{6}{|l|}{ PS } \\
\hline $0 / \mathrm{I}$ & 41.0 & \multirow{2}{*}{$<0.001$} & \multirow{2}{*}{4.2} & \multirow{2}{*}{0.001} & \multirow{2}{*}{$2.6-6.8$} \\
\hline II & 8.0 & & & & \\
\hline \multicolumn{6}{|l|}{ Treatment } \\
\hline PDS & 46.0 & \multirow{2}{*}{0.047} & \multirow{2}{*}{1.5} & \multirow{2}{*}{0.017} & \multirow{2}{*}{$1.10-1.70$} \\
\hline IDS & 43.0 & & & & \\
\hline \multicolumn{6}{|c|}{ Cytoreduction* } \\
\hline Optimal & 49 & \multirow{2}{*}{0.8} & \multirow{2}{*}{ NA } & \multirow{2}{*}{ NA } & \multirow{2}{*}{ NA } \\
\hline Suboptimal & 47 & & & & \\
\hline \multicolumn{6}{|l|}{ Grades } \\
\hline $\mathrm{I} / \mathrm{II}$ & 52.4 & \multirow{2}{*}{$<0.001$} & \multirow{2}{*}{1.5} & \multirow{2}{*}{0.017} & \multirow{2}{*}{$1.1-1.7$} \\
\hline III & 46.4 & & & & \\
\hline
\end{tabular}

${ }^{*}$ Cytoreduction was not included in multivariate analysis as it was insignificant in univariate analysis. Abbreviations: CI; confidence interval; HR, hazards ratio; IDS, interval debulking surgery; NA, not applicable; OS, overall survival; PDS, primary debulking surgery; PS, performance status. 


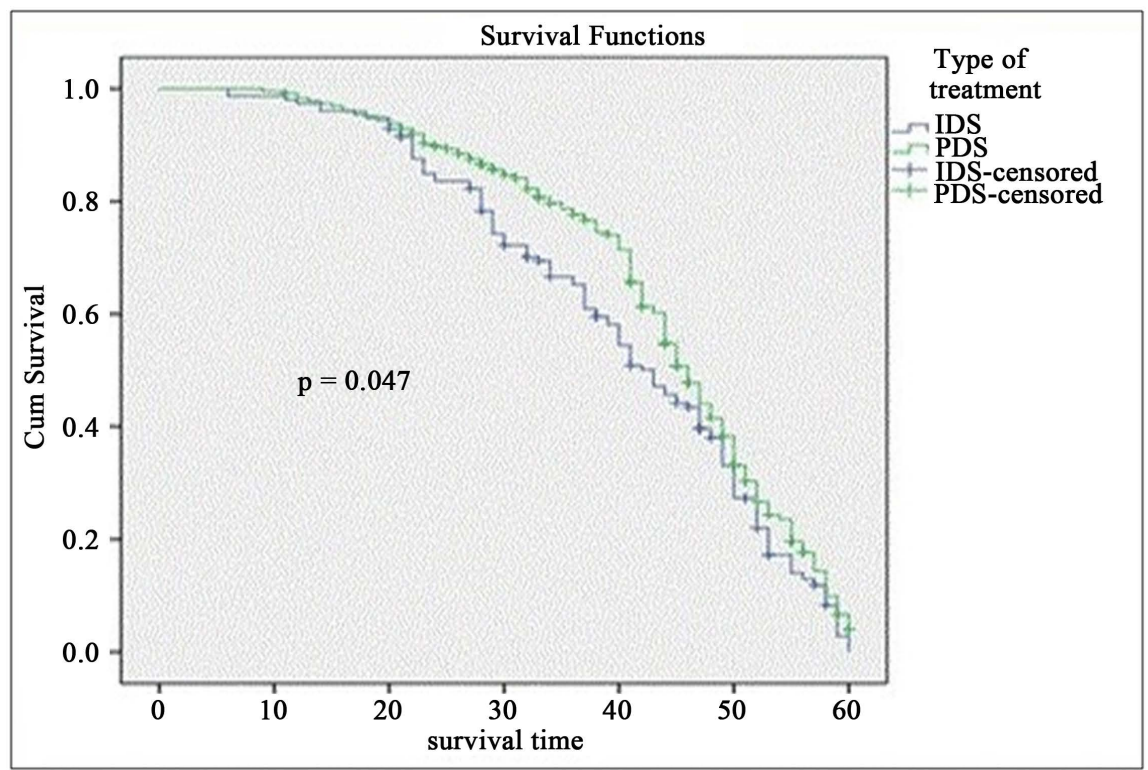

Figure 3. Kaplan-Meier analysis of OS in patients with stage III epithelial ovarian cancer.

Table 4. Subgroup survival analysis according to treatment modality.

\begin{tabular}{|c|c|c|c|c|c|c|c|c|}
\hline \multirow[b]{2}{*}{ Variable } & \multicolumn{4}{|c|}{ DFS } & \multicolumn{4}{|c|}{ OS } \\
\hline & $\begin{array}{l}\text { IDS Mean } \\
\text { Survival }\end{array}$ & $\begin{array}{c}\text { PDS Mean } \\
\text { Survival }\end{array}$ & $\begin{array}{c}\text { Survival } \\
\text { Ratio }\end{array}$ & $\mathrm{p}$ Value & $\begin{array}{c}\text { IDS Mean } \\
\text { Survival }\end{array}$ & $\begin{array}{c}\text { PDS Mean } \\
\text { Survival }\end{array}$ & $\begin{array}{c}\text { Survival } \\
\text { Ratio }\end{array}$ & $\mathrm{p}$ Value \\
\hline Age $\leq 60$ ys & 36 & 42.8 & 0.84 & $<0.001$ & 41.3 & 45.4 & 0.9 & 0.04 \\
\hline Age $\geq 60$ ys & 30 & 38.3 & 0.78 & 0.006 & 39.4 & 41.7 & 0.95 & 0.5 \\
\hline $\mathrm{PS}=0 / \mathrm{I}$ & 36.2 & 43.7 & 0.82 & $<0.001$ & 41.5 & 46 & 0.9 & 0.009 \\
\hline $\mathrm{PS}=\mathrm{II}$ & 18.5 & 7.8 & 2.3 & 0.007 & 33.7 & 16.2 & 2 & $<0.001$ \\
\hline Grade $=$ I/II & 37.7 & 42.6 & 0.88 & 0.006 & 36.8 & 44.3 & 0.83 & $<0.001$ \\
\hline Grade $=$ III & 30.4 & 39.7 & 0.84 & $<0.001$ & 44.3 & 43.7 & 1 & 0.27 \\
\hline $\begin{array}{c}\text { Optimal } \\
\text { Cytoreduction }\end{array}$ & 40 & 45.5 & 0.88 & 0.001 & 38.3 & 45 & 0.85 & $<0.001$ \\
\hline $\begin{array}{l}\text { Suboptimal } \\
\text { Cytoreduction }\end{array}$ & 23.7 & 34.7 & 0.68 & $<0.001$ & 44.8 & 42.3 & 1.05 & 0.14 \\
\hline
\end{tabular}

Abbreviations: DFS, disease-free survival; IDS, interval debulking surgery; OS, overall survival; PDS, primary debulking surgery; PS, performance status.

\section{Discussion}

In this study, primary debulking surgery (PDS) was better in OS and DFS than interval debulking surgery (IDS) for FIGO stage III epithelial ovarian carcinoma. In most of the previous studies, there is no consensus regarding this point which may be due to combining both FIGO stage III and IV as advanced ovarian cancer as well as the differences in the design of the studies [13]-[18]. Therefore, in our study, we evaluated FIGO stage III epithelial ovarian carcinoma separately that may explain more details about the outcome of this category of these patients. 
In univariate and multivariate analysis to show the risk factors that may affect OS and DFS, we found that treatment modality was a significant and independent prognostic factor for disease-free survival (DFS) and overall survival (OS) which are significantly better after PDS compared to IDS. We even found that DFS was significantly dropped in patients with bad performance status and suboptimal cytoreduction. Also, OS was significantly reduced in patients with bad performance status, older age, and high-grade tumors.

Regarding subgroup analysis, only the subgroup with performance status II got significant benefit from IDS in terms of DFS (DFS of IDS/PDS was 18.5/7.8 months, $\mathrm{p}=0.007)$. Again, the only subgroup that got benefit in OS from IDS was patients with performance status II (OS of IDS/PDS was 33.7/16 months, $\mathrm{p}=$ 0.000). In this subgroup, OS survival nearly doubled in comparison to PDS. This may be explained that neoadjuvant chemotherapy can resolve pleural effusions and ascites and improve the patient's performance status prior to surgery [19] [20]. These effects may reflect the better outcome of IDS in this subgroup. There have been reports of subjective improvements in the sense of well being and quality of life [21] [22]. It can decrease tumor volume and increase resectability. Thus, patients may have less intraoperative blood loss, shorter operative times, less intensive care unit admissions, and shorter length of hospital stay [23] [24] [25]. These issues are particularly important for patients with medical co-morbidities and a low probability of cure. Therefore, NACT may benefit a selected group of patients with low-performance status.

Optimal cytoreduction is a critical prognostic factor for prolonged survival, whether it is performed before or after chemotherapy. In this study, the overall DFS was significantly reduced from 45 months after optimal cytoreduction to 29 months after suboptimal cytoreduction $(\mathrm{p}=0.000)$. However, OS was not significantly different between the two groups (Table 2). Gao et al. demonstrated that patients debulked to no residual disease (RD) after PDS had the longest DFS and OS. For patients debulked to $\mathrm{RD}<1 \mathrm{~cm}$ or to $\mathrm{RD} \geq 1 \mathrm{~cm}$ after PDS, their prognosis had no significant differences. Moreover, after NACT, patients who were debulked to no RD after IDS had a significantly lower DFS and OS compared to patients with no RD after PDS [26]. These results agree with our results regarding the optimal debulking.

In the 2018 SGO (Society of Gynecologic Oncology) annual meeting on women's cancer, Dr. Beryl suggested a new prospective. If R0 was not attained, low volume disease confined to single anatomic locations $(\leq 1 \mathrm{~cm}-\mathrm{SL})$ may be an alternative [27]. The study showed that patients with $\mathrm{RD} \leq 1 \mathrm{~cm}$ involving multiple anatomic locations ( $\leq 1 \mathrm{~cm}-\mathrm{ML}$ ) had similar outcomes to suboptimal debulked $(\mathrm{RD}>1 \mathrm{~cm})$ patients. Moving beyond complete cytoreduction, low volume RD may be another option for consideration.

With the improvement of surgical techniques, several previous "unresectable" tumors could now be removed meticulously. In our series, we confirmed that the value of diagnostic laparoscopy for resectability assessment; patients of PDS arm who underwent laparoscopy before surgery showed a significantly higher rate of 
complete debulking compared to patients who did not undergo laparoscopy (data not shown). In our opinion, laparoscopic assessment before PDS or IDS should become one of the fundamental diagnostic steps to drive treatment decision.

One of the limitations of the present study is its retrospective nature.

\section{Conclusion}

This study showed that PDS resulted in a better disease-free survival and overall survival than IDS. Moreover, OS and DFS have significantly dropped in elderly patients, patients with bad performance status, suboptimal cytoreduction as well as high grade and undifferntated tumors. Therefore, patients selection should be considered.

\section{Conflicts of Interest}

Authors have no conflict of interests to declare.

\section{Ethical Considerations}

Approval for this study was obtained from our Ethical Committee.

\section{References}

[1] Jemel, A., Tiwari, R.C., Murray, T., et al. (2004) Cancer Statistics, 2004. CA: A Cancer Journal for Clinicians, 54, 8-29. https://doi.org/10.3322/canjclin.54.1.8

[2] Harries, M. and Gore, M. (2002) Part I: Chemotherapy for Epithelial Ovarian Cancer Treatment at First Diagnosis. The Lancet Oncology, 3, 529-536. https://doi.org/10.1016/S1470-2045(02)00846-X

[3] Griffiths, C.T., Parker, L.M. and Fuller, A.F. (1979) Role of Cytoreductive Surgical Treatment in the Management of Advanced Ovarian Cancer. Cancer Treatment Reviews, 63, 235-240.

[4] Hoskins, W.J. (1993) Surgical Staging and Cytoreductive Surgery of Epithelial Ovarian Cancer. Cancer, 71, 1534-1540. https://doi.org/10.1002/cncr.2820710420

[5] Hoskins, W.J., Bundy, B.N., Thigpen, J.T. and Omura, G.A. (1992) The Influence of Cytoreductive Surgery on Recurrence-Free Interval and Survival in Small-Volume Stage III Epithelial Ovarian Cancer: A Gynecologic Oncology Group Study. Gynecologic Oncology, 47, 159-166. https://doi.org/10.1016/0090-8258(92)90100-W

[6] Chi, D.S., Musa, F., Dao, F., Zivanovic, O., Sonoda, Y., Leitao, M.M., Levine, D.A., Gardner, G.J., Abu-Rustum, N.R. and Barakat, R.R. (2012) An Analysis of Patients with Bulky Advanced Stage Ovarian, Tubal, and Peritoneal Carcinoma Treated with Primary Debulking Surgery (PDS) during an Identical Time Period as the Randomized EORTC-NCIC Trial of PDS vs Neoadjuvant Chemotherapy (NACT). Gynecologic Oncology, 124, 10-14. https://doi.org/10.1016/j.ygyno.2011.08.014

[7] Vergote, I., De Wever, I., Tjalma, W., Van Gramberen, M., Decloedt, J. and van Dam, P. (1998) Neoadjuvant Chemotherapy or Primary Debulking Surgery in Advanced Ovarian Carcinoma: A Retrospective Analysis of 285 Patients. Gynecologic Oncology, 71, 431-436. https://doi.org/10.1006/gyno.1998.5213

[8] Inciura, A., Simavicius, A., Juozaityte, E., Kurtinaitis, J., Nadisauskiene, R., Svedas, E. and Kajenas, S. (2006) Comparison of Adjuvant and Neoadjuvant Chemotherapy 
in the Management of Advanced Ovarian Cancer: A Retrospective Study of $574 \mathrm{~Pa}$ tients. BMC Cancer, 6, 153. https://doi.org/10.1186/1471-2407-6-153

[9] Schwartz, P.E., Rutherford, T.J., Chambers, J.T., Kohorn, E.I. and Thiel, R.P. (1999) Neoadjuvant Chemotherapy for Advanced Ovarian Cancer Long-Term Survival. Gynecologic Oncology, 72, 93-99. https://doi.org/10.1006/gyno.1998.5236

[10] Morice, P., Dubernard, G., Rey, A., Atallah, D., Pautier, P., Pomel, C., Lhomme, C., Duvillard, P. and Castaigne, D. (2003) Results of Interval Debulking Surgery Compared with Primary Debulking Surgery in Advanced Stage Ovarian Cancer. Journal of the American College of Surgeons, 197, 955-963. https://doi.org/10.1016/j.jamcollsurg.2003.06.004

[11] Sehouli, J., Savvatis, K., Braicu, E.I., Schmidt, S.C., Lichtenegger, W. and Fotopoulou, C. (2010) Primary versus Interval Debulking Surgery in Advanced Ovarian Cancer: Results from a Systematic Single-Center Analysis. International Journal of Gynecological Cancer, 20, 1331-1340.

[12] Bristow, R.E. and Chi, D.S. (2006) Platinum-Based Neoadjuvant Chemotherapy and Interval Surgical Cytoreduction for Advanced Ovarian Cancer: A Meta-Analysis. Gynecologic Oncology, 103, 1070-1076. https://doi.org/10.1016/j.ygyno.2006.06.025

[13] Coleridge, S.L., Bryant, A., Lyons, T.J., Goodall, R.J., Kehoe, S. and Morrison, J. (2019) Chemotherapy versus Surgery for Initial Treatment in Advanced Ovarian Epithelial Cancer. Cochrane Database of Systematic Reviews, CD005343. https://doi.org/10.1002/14651858.CD005343.pub4

[14] Kobal, B., Noventa, M., Cvjeticanin, B., Barbic, M., Meglic, L., Herzog, M., et al. (2018) Primary Debulking Surgery versus Primary Neoadjuvant Chemotherapy for High Grade Advanced Stage Ovarian Cancer: Comparison of Survivals. Radiology and Oncology, 52, 307-319. https://doi.org/10.2478/raon-2018-0030

[15] Yang, L., Zhang, B., Xing, G., Du, J., Yang, B., Yuan, Q., et al. (2017) Neoadjuvant Chemotherapy versus Primary Debulking Surgery in Advanced Epithelial Ovarian Cancer: A Meta-Analysis of Peri-Operative Outcome. PLOS ONE, 12, e0186725. https://doi.org/10.1371/journal.pone.0186725

[16] Xiao, Y., Xie, S., Zhang, N., Wang, J., Lv, C., Guo, J., et al. (2018) Platinum-Based Neoadjuvant Chemotherapy versus Primary Surgery in Ovarian Carcinoma International Federation of Gynecology and Obstetrics Stages IIIc and IV: A Systematic Review and Meta-Analysis. Gynecologic and Obstetric Investigation, 83, 209-219. https://doi.org/10.1159/000485618

[17] May, T., Comeau, R., Sun, P., Kotsopoulos, J., Narod, S.A., Rosen, B., et al. (2017) A Comparison of Survival Outcomes in Advanced Serous Ovarian Cancer Patients Treated with Primary Debulking Surgery versus Neoadjuvant Chemotherapy. International Journal of Gynecological Cancer, 27, 668-674. https://doi.org/10.1097/IGC.0000000000000946

[18] Siesto, G., Cavina, R., Romano, F. and Vitobello, D. (2018) Primary Debulking Surgery versus Neoadjuvant Chemotherapy in Advanced Epithelial Ovarian Cancer: A Propensity-Matched Analysis. American Journal of Clinical Oncology, 41, 280-285.

[19] Kayikcioglu, F., Kose, M.F., Boran, N., Caliskan, E. and Tulunay, G. (2001) Neoadjuvant Chemotherapy or Primary Surgery in Advanced Epithelial Ovarian Carcinoma. International Journal of Gynecological Cancer, 11, 466-470. https://doi.org/10.1046/j.1525-1438.2001.01064.x

[20] Surwit, E., Childers, J., Atlas, I., Nour, M., Hatch, K., Hallum, A. and Alberts, D. (1996) Neoadjuvant Chemotherapy for Advanced Ovarian Cancer. International Journal of Gynecological Cancer, 6, 356-361. 
https://doi.org/10.1046/j.1525-1438.1996.06050356.x

[21] Chambers, J.T., Chambers, S.K., Voynick, I.M. and Schwartz, P.E. (1990) Neo-Adjuvant Chemotherapy in Stage X Ovarian Carcinoma. Gynecologic Oncology, 37, 327-331. https://doi.org/10.1016/0090-8258(90)90361-N

[22] Schwartz, P.E., Chambers, J.T. and Makuch, R. (1994) Neoadjuvant Chemotherapy for Advanced Ovarian Cancer. Gynecologic Oncology, 53, 33-37. https://doi.org/10.1006/gyno.1994.1083

[23] Hoskins, W.J. (1993) Surgical Staging and Cytoreductive Surgery of Epithelial Ovarian Cancer. Cancer, 71, 1534-1540. https://doi.org/10.1002/cncr.2820710420

[24] Jacob, J.H., Gershenson, D.M., Morris, M., Copeland, L.J., Burke, T.W. and Wharton, J.T. (1991) Neoadjuvant Chemotherapy and Interval Debulking for Advanced Epithelial Ovarian Cancer. Gynecologic Oncology, 42, 146-150. https://doi.org/10.1016/0090-8258(91)90335-3

[25] Schwartz, P.E., Rutherford, T.J., Chambers, J.T., Kohorn, E.I. and Thiel, R.P. (1999) Neoadjuvant Chemotherapy for Advanced Ovarian Cancer: Long-Term Survival. Obstetrical \& Gynecological Survey, 54, 379-381. https://doi.org/10.1097/00006254-199906000-00016

[26] Gao, Y., Li, Y., Zhang, J., Han, L.H., Zhang, K. and Guo, H. (2019) Evaluating the Benefits of Chunyu Neoadjuvant Chemotherapy for Advanced Epithelial Ovarian Cancer: A Retrospective Study. Journal of Ovarian Research, 12, 85. https://doi.org/10.1186/s13048-019-0562-9

[27] Manning-Geist, B.L., Hicks-Courant, K., Gockley, A.A., Clark, R.M., Del Carmen, M., Growdon, W.B., et al. (2018) Moving beyond "Complete Surgical Resection" and "Optimal": Is Low-Volume Residual Disease Another Option for Primary Debulking Surgery? Gynecologic Oncology, 150, 233-238. https://doi.org/10.1016/j.ygyno.2018.06.015

\section{Abbreviation}

AUC5: Area under the Curve 5.

CIs: Confidence Intervals.

CONSORT: Consolidated Standards of Reporting Trials.

DFS: Disease-Free Survival.

FIGO: International Federation of Gynecology and Obstetrics.

HRs: Hazards Ratios.

IDS: Interval Debulking Surgery.

NA: Not Applicable.

NACT: Neoadjuvant Chemotherapy.

OC: Ovarian Cancer.

OS: Overall Survival.

PDS: Primary Debulking Surgery.

PS: Performance Status.

RD: Residual Disease.

SGO: Society of Gynecologic Oncology. 